\title{
Two-port laparoscopic appendectomy with the help of a needle grasper: better cosmetic results and fewer trocars than conventional laparoscopic appendectomy
}

\author{
Turgut Donmez ${ }^{1}$, Oguzhan Sunamak ${ }^{2}$, Sina Ferahman ${ }^{3}$, Server Sezgin Uludag ${ }^{3}$, Dogan Yildirim ${ }^{4}$, Adnan Hut $^{4}$ \\ ${ }^{1}$ Department of General Surgery, Lutfiye Nuri Burat State Hospital, Istanbul, Turkey \\ 2Department of General Surgery, Haydarpasa Numune Training and Research Hospital, Istanbul, Turkey \\ ${ }^{3}$ Department of General Surgery, Istanbul University Cerrahpasa Medical Faculty, Istanbul, Turkey \\ ${ }^{4}$ Department of General Surgery, Haseki Training and Research Hospital, Istanbul, Turkey
}

Videosurgery Miniinv 2016; 11 (2): 105-110

DOI: 10.5114/wiitm.2016.60504

\begin{abstract}
Introduction: The two-port laparoscopic appendectomy technique (TPLA) lays between the conventional three-port trocar procedure and single-port laparoscopic appendectomy surgery. During TPLA, the appendix is suspended with stitches, resulting in perforation risk and difficulty in exploration.

Aim: We used a needle grasper in TPLA to hang and manipulate the appendix.

Material and methods: Thirty-four patients (10 female, 24 male) who underwent TPLA between February 2015 and November 2015 were analyzed retrospectively for patient demographics, duration of operation, laparotomy or conventional laparoscopy necessity, drain use, complications, and hospital stay periods. The needle grasper was inserted at the right under the abdominal quadrant (McBurney point) without an incision to hang and manipulate the appendix.

Results: The mean age was $25.19 \pm 8.464$ years; the mean body mass index (BMI) was $23.50 \pm 3.246 \mathrm{~kg} / \mathrm{m}^{2}$. ASA scores were 1 and 2. The operations were completed without any additional trocar in 34 patients. The mean operation time was $57.03 \pm 3.814 \mathrm{~min}$. There were no intraoperative complications in any patients. Three patients required a drain; all were discharged after drain removal. Thirty-one patients were discharged on the $1^{\text {st }}$ postoperative day; three patients with drains were discharged on the $2^{\text {nd }}$ day. The mean hospital stay period was $1.18 \pm 0.535$ days.

Conclusions: Using the needle grasper, the appendix was held and suspended and the mesoappendix was cauterized and skeletonized successfully in TPLA. Inserting a needle grasper into the abdominal cavity at the McBurney point to manipulate the appendix helps and does not leave a visible scar.
\end{abstract}

Key words: laparoscopic appendectomy, minimally invasive surgery, needle grasper.

\section{Introduction}

Laparoscopic appendectomy (LA) was recently accepted as the gold standard procedure for complicated or uncomplicated acute appendicitis [1]. Better cosmetic results, less postoperative pain, less postoperative infection, decreased length of hospi- talization, and early recovery are the advantages of LA [2, 3]. The recently developed single-port technique advanced the level of minimally invasive surgery. Most of the single-port appendectomy studies have not revealed any advantages other than technical feasibility and cosmetic results. In spite of the 
advantages of the reduced port number, increased incision diameter, and wider fascia defect, more postoperative pain and longer operation times are among the disadvantages of the single-port technique. Another disadvantage of the single-port technique is higher operation costs [3-6]. Two-port LA (TPLA) is a minimally invasive surgical procedure and results in shorter incisions, less postoperative pain, and a good cosmetic result. There are many studies related to this technique $[7,8]$.

We performed TPLA with the help of an additional needle grasper (percutaneous organ holder device). This method, due to having fewer ports than the three-port LA and less surgical trauma, resulted in better cosmetic results.

\section{Aim}

This study presents our experience with the needle grasper in two-port laparoscopic appendectomy.

\section{Material and methods}

This retrospective study was performed at a state hospital with 100 beds in Istanbul, between February 2015 and November 2015, with two surgeons who are experienced in laparoscopic surgery. We retrospectively analyzed the 34 patients with acute appendicitis who underwent LA. Patients were diag-
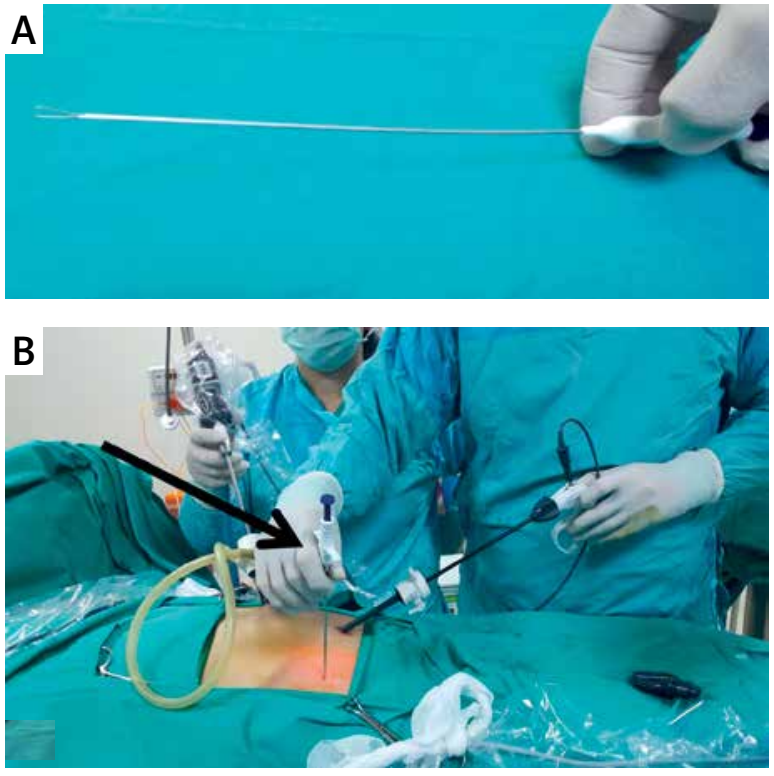

Photo 1. A - Needle grasper (percutaneous organ holder device), $\mathbf{B}$ - abdominal entry point of the needle grasper nosed with acute appendicitis by abdominal ultrasonography (25 patients) or computed tomography (9 patients). The chosen LA techniques were the surgeon's own choice. Thirty-two patients who underwent TPLA were included. Cases of complicated appendicitis, such as generalized peritonitis or plastron formation, were excluded. Informed consent forms were obtained from all patients before the surgery.

Age, sex, height, weight, body mass index (BMI), and co-morbid diseases of the patients were recorded. Duration of operation, complications, hospital stay period, and enteral feeding initiation time were recorded. Postoperative complications, such as wound infections, stump leakage, ileus, and intra-abdominal abscess, were recorded. The needle grasper scar was checked at the $20^{\text {th }}$ and $30^{\text {th }}$ postoperative days and recorded.

This study was approved by the Ethics Committee of Haseki Research and Training Hospital on 11.11.2015 with approval number 274 .

\section{Surgical method}

Operations were performed by a left-positioned surgeon and an assistant (scopist). All patients were given general anesthesia. In the TPLA, a 1-cm skin incision under the umbilicus was performed and the intra-abdominal cavity was accessed with a Veress needle. For pneumoperitoneum, 14-mm Hg of $\mathrm{CO}_{2}$ pressure was generated and a $10-\mathrm{mm}$ trocar was inserted into the intraperitoneal cavity. A $0^{\circ}, 5 \mathrm{~mm}$ optical camera was inserted through the umbilical trocar and the $5 \mathrm{~mm}$ trocar entered through the suprapubic area. Patients were positioned in the Trendelenburg position with a 15-degree angle to the left. Diagnostic exploration was performed and acute appendicitis was verified. A needle grasper (Percutaneous Organ Holder Device, Suture Grasper Closure Device, Mediflex Surgical Products, Islandia, NY, USA) was entered through the McBurney point (Photos 1 A, B). The appendix was held with the needle grasper. Similarly to an endograsper, the appendix was manipulated easily and successfully (Photo 2 A). The mesoappendix was cauterized and cut with a LigaSure instrument (Covidien, Boulder, CO). An endoloop was inserted through the 5-mm trocar and passed around the tip of the appendix to the radix and tightened (Photo $2 \mathrm{~B}$ ). Appendectomy was performed. The optical camera was inserted through the $5-\mathrm{mm}$ suprapubic trocar and the endobag was inserted through the $10-\mathrm{mm}$ umbilical trocar; the 

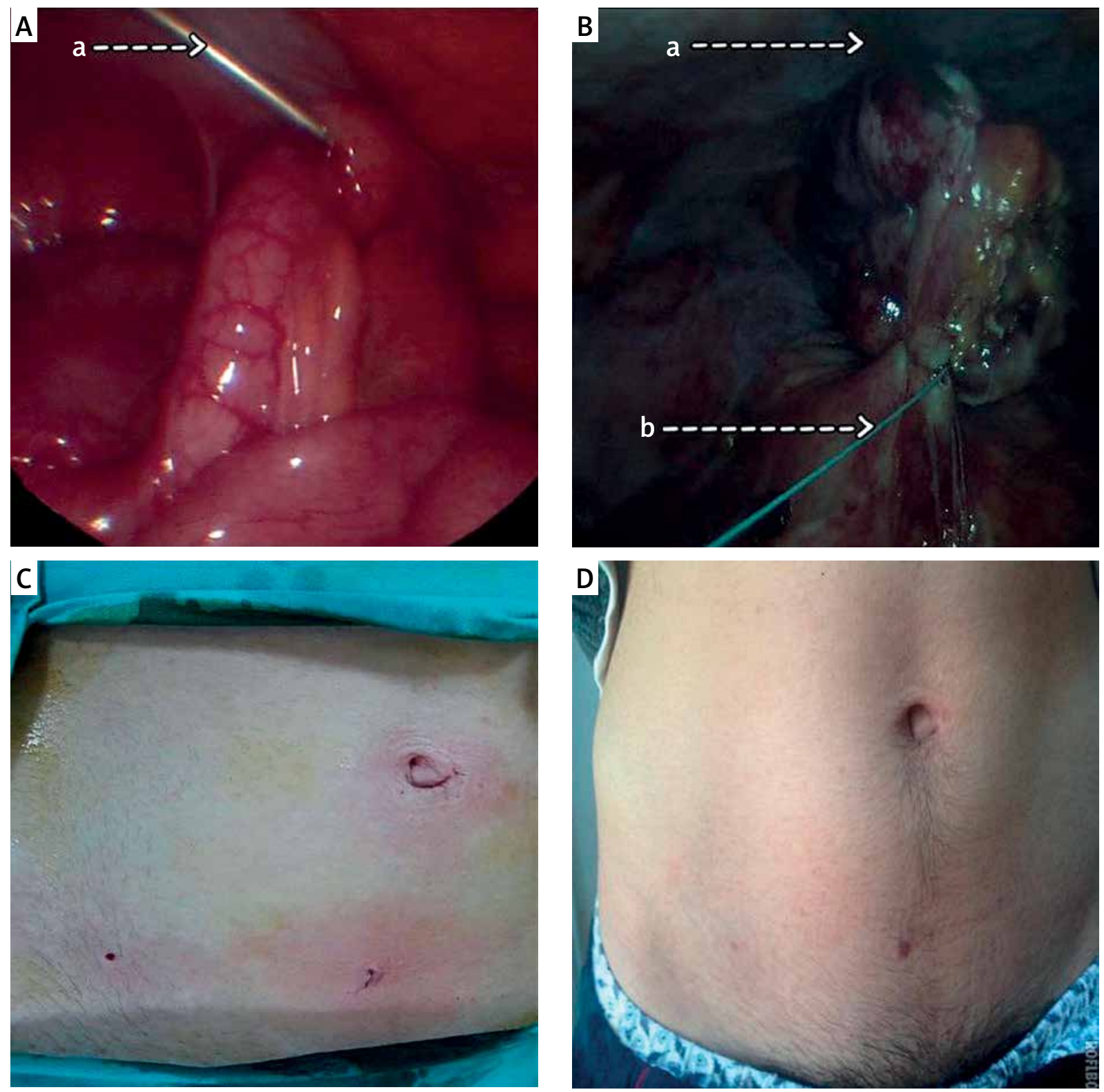

Photo 2. A - Holding of appendix by needle grasper, B - a: Needle grasper device, b: Endoloop, C - immediate image of the abdomen, $\mathbf{D}$ - image of the abdomen, 20 days after the operation

surgical specimen was put into the endobag and taken out of the abdomen. The fascia was closed with 2/0 vicryl and the skin was closed with 4/0 intracutaneous vicryl sutures.

\section{Results}

Thirty-four (10 female, 24 male) patients were included. The mean age was 25.19 \pm 8.464 years (min: 16; max: 48), and the mean BMI was $22.49 \pm 2.951 \mathrm{~kg} / \mathrm{m}^{2}$ (min: 19.6; max: 43.25). Thirty-two patients were
ASA 1 and 2 patients were ASA 2. Two patients had a perforated plastron appendicitis upon laparoscopic exploration and were excluded.

The operations were completed without any additional trocar or complications in the remaining 34 patients. The mean operation time was 57.03 $\pm 3.814 \mathrm{~min}$ (minimum: $48 \mathrm{~min}$, maximum: $68 \mathrm{~min}$ ).

One patient who underwent TPLA had a postoperative infection. We observed the infection at the periumbilical incision region. The patient recovered with medical treatment. 
Three patients required Hemovac drain placement into the cul-de-sac or Douglas cavity due to minor blood leakage from the mesoappendix. All the patients were discharged after removal of the drain. Thirty-one patients were discharged on the $1^{\text {st }}$ postoperative day. Three patients with drains were discharged on the $2^{\text {nd }}$ postoperative day. The mean hospitalization period was $1.18 \pm 0.535$ days (min: 1 day, max: 2 days). The cosmetic results of the needle grasper entry port were very impressive, both during the early postoperative period and during the 20-day follow-up.

\section{Discussion}

Technological progressed has opened a new era in medicine. As a result of laparoscopic improvements, operations have quickly progressed to SILS ports and robotic surgery. Many researchers have developed different techniques for natural orifice transluminal endoscopic surgery, and there are reported LAs via a vaginal approach [9, 10]. However, there are many risks to these procedures. Umbilical access laparoscopic procedures are a standardized methodology for laparoscopic intra-abdominal surgery. LAs are usually performed via umbilical access. There are many techniques for LAs: standard multiport LA, single-port LA, and TPLA are some such transumbilical methods. Standard LA is a surgical procedure using 3 ports. SILS port LA is a surgical procedure using a single port that has 3 or 4 internal lumens. In the SILS port procedure, a $2-\mathrm{cm}$ incision is necessary, and this may result in more postoperative pain and infection. Many studies have reported postoperative pain occurring more often after the SILS port technique [11-13]. In one study, Mayer et al. reported less postoperative pain in the SILS group [14].

In TPLA, better angulation, compared to the SILS port and conventional LA operation, can be easily achieved. In this technique, the percutaneous organ holder device may function like a grasper [15]. With the help of this device, postoperative pain and scarring may be minimized.

The most important point for SILS port appendectomy and TPLA is the proper position for mesoappendix dissection. During the dissection of the mesoappendix, in order to cauterize and cut the appendicular artery, the appendix should be tracked. There are many defined techniques to achieve this position. Roberts defined the "puppeteer tech- nique," a suture outgoing from the right iliac fossa and suspended by the left hand of the surgeon [16]. The procedures were performed successfully in 13 of 14 cases in this puppeteer technique report, and they reported less postoperative pain and better cosmetic results.

Yeung used a string loop, which was passed through an intravenous catheter at McBurney's point, to hang the appendix. In this technique, they used an intravenous catheter and inserted the needle into the abdomen, suspended the appendix against the abdominal wall, and performed the appendectomy in 14 cases without complications. They stated that it was especially useful in cases with inflamed appendixes, and the McBurney point is ideal for decreasing infections and complications; there was no scarring at the catheter port [17].

Ate et al. fixed the mesoappendix to the right iliac fossa with a transabdominal suture in single-port LAs [18]. They reported better cosmetic results with this technique and had fewer tools in the abdomen.

In another study of TPLA, the appendix was fixed to the abdominal wall via a suture loop on the anterior abdominal wall in the right lower quadrant, which was used as an axle [19]. A suture from the appendix to the outside of the abdomen through the port was used to handle the appendix.

In our study, inserting the needle grasper (a percutaneous organ holder device) at the McBurney point, which has been proven to be a safe point by many studies, provided a means of holding and suspending the appendix. The surgeon could adjust the position of the appendix by the needle grasper in his left hand. The mesoappendix was cauterized by the LigaSure instrument through the suprapubic trocar and the appendix was skeletonized. The appendix was sutured at the radix with an endoloop through the suprapubic trocar and appendectomy was performed. We did not encounter complications, such as appendix perforation or organ perforation, due to the use of the needle grasper. We did not observe postoperative pain or infection at the entrance point of the needle grasper. The diameter of the device was $2.1 \mathrm{~mm}$, and there was no need for an extra incision for the needle's insertion. The reason for using the McBurney point was its safety and because that point is an ideal region for infection sparing [16-19]. With the help of camera light transillumination, insertion into the abdomen was easier and safer. Using the needle grasper, the appendix was held 
and suspended and the mesoappendix was cauterized with the LigaSure instrument and skeletonized successfully. Decreased postoperative pain and easy suspension of the appendix were the advantages of this technique. We think that this technique can be used in single-port LAs.

The immediate postoperative view of the entrance point is shown in Photo $2 \mathrm{C}$. We did not observe scar tissue at the entrance point at the $20^{\text {th }}$ postoperative day (Photo $2 \mathrm{D}$ ). All procedures were performed successfully. In TPLA, we observed 1 wound infection at the periumbilical region that was easily treated.

Following pneumoperitoneum, intra-abdominal pathologies could be seen easily in both groups. In our series, we could examine the intestines, fallopian tubes, and ovaries easily before appendectomy. In one patient, we observed a perforated Meckel diverticulum and we performed diverticulectomy with conventional laparoscopic techniques. This patient was excluded from the study.

Conversion of a technique should be performed without any hesitation. We do not think of the conversion of a technique as a complication or failure. This entity is very important and an obligation in emergency surgical operations. In order to achieve better vision, the number of ports should be increased, if necessary. Inserting a suprapubic trocar may provide better manipulation for treating retrocaecal, purulent, or gangrenous acute appendicitis. The suprapubic approach makes dissection easier and it is a good point in case drainage is necessary.

The decreased number of trocars may result in better cosmetic results; however, there is no standardized scoring system for wound infection in order to evaluate the cosmetic results. In our study, we did not observe any obvious scar tissue at the entrance point of the needle grasper at the $20^{\text {th }}$ postoperative day. All patients were satisfied with the cosmetic results.

\section{Conclusions}

We observed in our study that the needle grasper can be helpful not only in appendectomy, but also in different kinds of minimally invasive laparoscopic operations.

As a result, TPLA with the help of a needle grasper can give good results. By using this technique, the number of ports used can be decreased and better cosmetic results may be achieved.
The benefits of these new techniques need to be assessed in randomized clinical trials.

\section{Conflict of interest}

The authors declare no conflict of interest.

\section{References}

1. Tiwari MM, Reynoso JF, Tsang AW, Oleynikov D. Comparison of outcomes of laparoscopic and open appendectomy in management of uncomplicated and complicated appendicitis. Ann Surg 2011; 254: 927-32.

2. Taguchi Y, Komatsu S, Sakamoto E, et al. Laparoscopic versus open surgery for complicated appendicitis in adults: a randomized controlled trial. Surg Endosc 2016; 30: 1705-12.

3. Lee J, Baek J, Kim W. Laparoscopic transumbilical single-port appendectomy: initial experience and comparison with threeport appendectomy. Surg Laparosc Endosc Percutan Tech 2010; 20: 100-3.

4. Lee YS, Kim JH, Moon EJ, et al. Comparative study on surgical outcomes and operative costs of transumbilical single-port laparoscopic appendectomy versus conventional laparoscopic appendectomy in adult patients. Surg Laparosc Endosc Percutan Tech 2009; 19: 493-6.

5. Xu AM, Huang L, Li TJ. Single-incision versus three port laparoscopic appendectomy for acute appendicitis: systematic review and meta-analysis of randomized controlled trials. Surg Endosc 2015; 29: 822-43.

6. Zhang Z, Wang Y, Liu R, et al. Suprapubic single-incision versus conventional laparoscopic appendectomy. J Surg Res 2016; 200: 131-8.

7. Olijnyk JG, Pretto GG, da Costa Filho OP, et al. Two-port laparoscopic appendectomy as transition to laparoendoscopic single site surgery. J Minim Access Surg 2014; 10: 23-6.

8. Gołębiewski A, Losin M, Murawski M, et al. One, two or three port appendectomy - a rational approach. Videosurgery Miniinv 2013; 8: 226-31.

9. Thomson JE, Kruger D, Jann-Kruger C, et al. Laparoscopic versus open surgery for compilated appendicitis: a randomized controlled trial to prove safety. Surg Endosc 2015; 29: 2027-32.

10. Bernhardt J, Steffen H, Schneider-Koriath S, Ludwig K. Clinical NOTES appendectomy study: comparison of transvaginal NOTES appendectomy in hybrid technique with laparoscopic appendectomy. Int J Colorectal Dis 2015; 30: 259-67.

11. Kim HO, Yoo CH, Lee SR, et al. Pain after laparoscopic appendectomy: a comparison of transumbilical single-port and conventional laparoscopic surgery. J Korean Surg Soc 2012; 82: 172-8.

12. Kang J, Bae BN, Gwak G, et al. Comparative study of a single-incision laparoscopic and a conventional laparoscopic appendectomy for the treatment of acute appendicitis. J Korean Soc Coloproctol 2012; 28: 304-8.

13. Vilallonga R, Barbaros U, Nada A, et al. Single-port transumbilical laparoscopic appendectomy: a preliminary multicentric comparative study in 87 patients with acute appendicitis. Minim Invasive Surg 2012; 2012: 492409. 
14. Mayer S, Werner A, Wachowiak R, et al. Single-incision multiport laparoscopy does not cause more pain than conventional laparoscopy: a prospective evaluation in children undergoing appendectomy. J Laparoendosc Adv Surg Tech A 2011; 21: 753-6.

15. Gulpinar K, Ozdemir S, Ozis SE, et al. Single incision laparoscopic cholecystectomy by using a $2 \mathrm{~mm}$ a traumatic grasper without trocar. HPB Surg 2011; 2011: 761315.

16. Roberts KE. True single-port appendectomy: first experience with the "puppeteer technique". Surg Endosc 2009; 23: 1825-30.

17. Yeung GH. The intravenous catheter at the McBurney's point. Surg Laparosc Endosc 1999; 9: 45-8.

18. Ate O, Hakgüder G, Olguner M, Akgür FM. Single-port laparoscopic appendectomy conducted intracorporeally with the aid of a transabdominal sling suture. J Pediatr Surg 2007; 42: 1071-4.

19. Panait L, Bell RL, Duffy AJ, Roberts KE. Two-port laparoscopic appendectomy: minimizing the minimally invasive approach. J Surg Res 2009; 153: 167-71.

Received: 19.04 .2016 , accepted: 30.05 .2016 